\title{
A NEW APPROACH TO MINIMISING BINARY QUARTICS AND TERNARY CUBICS
}

\author{
TOM FISHER
}

\begin{abstract}
We prove a theorem on the minimisation of genus one curves, generalising work of Birch and Swinnerton-Dyer [3], Cremona and Serf [5], [13] and Cremona and Stoll [6], [7]. The advantage of our approach over earlier methods is that we do not need to treat residue characteristics 2 and 3 as special cases.
\end{abstract}

\section{Invariants}

Let $C$ be a smooth curve of genus one defined over a field $K$, and let $D$ be a $K$-rational divisor on $C$ of degree $n \geq 2$. We write $[D]$ for the linear equivalence class of $D$.

1.1. Binary quartics. If $n=2$ then we pick $x, y \in K(C)$ such that $\mathcal{L}(D)$ and $\mathcal{L}(2 D)$ have bases $1, x$ and $1, x, x^{2}, y$. The 9 elements $1, x, x^{2}, y, x^{3}, x y, x^{4}, x^{2} y, y^{2}$ in the 8 dimensional vector space $\mathcal{L}(4 D)$ satisfy a linear dependence relation. Furthermore the coefficient of $y^{2}$ is non-zero. We deduce that the pair $(C,[D])$ has an equation

$$
y^{2}+\left(\alpha_{0} x^{2}+\alpha_{1} x+\alpha_{2}\right) y=a x^{4}+b x^{3}+c x^{2}+d x+e .
$$

If $\operatorname{char}(K) \neq 2$ then we may complete the square so that $\alpha_{0}=\alpha_{1}=\alpha_{2}=0$. The classical invariants of the binary quartic

$$
f(x, z)=a x^{4}+b x^{3} z+c x^{2} z^{2}+d x z^{3}+e z^{4}
$$

are

$$
\begin{aligned}
& I=12 a e-3 b d+c^{2}, \\
& J=72 a c e-27 a d^{2}-27 b^{2} e+9 b c d-2 c^{3} .
\end{aligned}
$$

We define the invariants $c_{4}$ and $c_{6}$ of (1) by taking $c_{4}=2^{4} I$ and $c_{6}=2^{5} J$ in the case $\alpha_{0}=\alpha_{1}=\alpha_{2}=0$. We then extend to the general case by demanding that $c_{4}$ and $c_{6}$ are preserved by all changes of co-ordinates of the form $y \mapsto y+r_{0} x^{2}+r_{1} x+r_{2}$. In addition we put $\Delta=\left(c_{4}^{3}-c_{6}^{2}\right) / 1728$. We find that $c_{4}, c_{6}$ and $\Delta$ are primitive integer coefficient polynomials in the indeterminates $\alpha_{0}, \alpha_{1}, \alpha_{2}, a, b, c, d, e$. This enables us to define the invariants $c_{4}, c_{6}$ and $\Delta$ in arbitrary characteristic. Moreover if we put

$$
\left(\alpha_{0}, \alpha_{1}, \alpha_{2}, a, b, c, d, e\right)=\left(0, a_{1}, a_{3}, 0,1, a_{2}, a_{4}, a_{6}\right)
$$

then our expressions for $c_{4}, c_{6}$ and $\Delta$ reduce to the standard formulae for an elliptic curve in Weierstrass form (recalled in $\S 4.1$ ). It is convenient to rewrite (1) as a homogeneous equation of degree 4 , where $x, z, y$ are assigned degrees $1,1,2$.

$$
y^{2}+\left(\alpha_{0} x^{2}+\alpha_{1} x z+\alpha_{2} z^{2}\right) y=a x^{4}+b x^{3} z+c x^{2} z^{2}+d x z^{3}+e z^{4} .
$$

By abuse of terminology we call (2) a binary quartic.

Received by the editors March, 152006. 
1.2. Ternary cubics. If $n=3$ then we pick a basis $x, y, z$ for $\mathcal{L}(D)$. Writing down 10 elements in the 9 dimensional vector space $\mathcal{L}(3 D)$ we deduce that $(C,[D])$ is defined by a ternary cubic

$$
\begin{aligned}
F(x, y, z)=a x^{3}+b y^{3}+c z^{3}+a_{2} x^{2} y+a_{3} x^{2} z \\
+b_{1} x y^{2}+b_{3} y^{2} z+c_{1} x z^{2}+c_{2} y z^{2}+m x y z .
\end{aligned}
$$

The Hessian of the ternary cubic $F\left(x_{1}, x_{2}, x_{3}\right)$ is

$$
H\left(x_{1}, x_{2}, x_{3}\right)=-1 / 2 \times \operatorname{det}\left(\frac{\partial^{2} F}{\partial x_{i} \partial x_{j}}\right)_{i, j=1,2,3} .
$$

The factor $-1 / 2$, although not standard, is a convenient choice. The Hessian of any linear combination of $F$ and $H$ again belongs to the pencil spanned by $F$ and $H$. Following [8], [12], the invariants $c_{4}$ and $c_{6}$ are determined by the relation

$$
H(\lambda F+\mu H)=3\left(c_{4} \lambda^{2} \mu+2 c_{6} \lambda \mu^{2}+c_{4}^{2} \mu^{3}\right) F+\left(\lambda^{3}-3 c_{4} \lambda \mu^{2}-2 c_{6} \mu^{3}\right) H .
$$

In addition we put $\Delta=\left(c_{4}^{3}-c_{6}^{2}\right) / 1728$. We find that $c_{4}, c_{6}$ and $\Delta$ are primitive integer coefficient polynomials in the indeterminates $a, b, c, a_{2}, a_{3}, b_{1}, b_{3}, c_{1}, c_{2}, m$. This enables us to define the invariants $c_{4}, c_{6}$ and $\Delta$ in arbitrary characteristic. Moreover if we put

$$
\left(a, b, c, a_{2}, a_{3}, b_{1}, b_{3}, c_{1}, c_{2}, m\right)=\left(-1,0,-a_{6}, 0,-a_{2}, 0,1,-a_{4}, a_{3}, a_{1}\right)
$$

then our expressions for $c_{4}, c_{6}$ and $\Delta$ reduce to the standard formulae for an elliptic curve in Weierstrass form (recalled in $§ 4.1$ ).

1.3. Computing the Jacobian. It was observed by Weil [16] that the invariants of a binary quartic may be used to compute its Jacobian. The generalisation to ternary cubics may be found in [1].

Theorem 1.1. Let $c_{4}, c_{6}$ and $\Delta$ be the invariants of a binary quartic, respectively a ternary cubic.

(a) The binary quartic, respectively ternary cubic, defines a smooth curve of genus one if and only if $\Delta \neq 0$.

(b) If char $(K) \neq 2,3$ then the Jacobian has Weierstrass equation

$$
y^{2}=x^{3}-27 c_{4} x-54 c_{6} .
$$

Notice that (3) has invariants $6^{4} c_{4}, 6^{6} c_{6}$ and $6^{12} \Delta$. As pointed out in [2] these formulae for the Jacobian can be improved by minimising at 2 and 3 . We give details in Appendix A.

\subsection{Equivalence of models.}

Definition 1.2. We say that a pair of binary quartics, respectively ternary cubics, are equivalent if they arise from the same pair $(C,[D])$.

More concretely, binary quartics are equivalent if they are related by making a substitution of the form

$$
\begin{aligned}
& x=\alpha x^{\prime}+\beta z^{\prime} \\
& z=\gamma x^{\prime}+\delta z^{\prime} \\
& y=\mu^{-1} y^{\prime}+r_{0} x^{2}+r_{1} x^{\prime} z^{\prime}+r_{2} z^{\prime 2}
\end{aligned}
$$


and then multiplying through by $\mu^{2}$. This transformation is denoted $[\mu, r, A]$ where $r=\left(r_{0}, r_{1}, r_{2}\right)$ and

$$
A=\left(\begin{array}{ll}
\alpha & \beta \\
\gamma & \delta
\end{array}\right) .
$$

Similarly, ternary cubics are equivalent if they are related by making a substitution of the form

$$
\left(\begin{array}{l}
x \\
y \\
z
\end{array}\right)=A\left(\begin{array}{l}
x^{\prime} \\
y^{\prime} \\
z^{\prime}
\end{array}\right)
$$

and then multiplying through by $\mu$. This transformation is denoted $[\mu, A]$. In both cases it is to be understood that $\mu \operatorname{det} A \neq 0$. Putting $\lambda=\mu \operatorname{det} A$ we find (again in both cases) that $c_{4}^{\prime}=\lambda^{4} c_{4}, c_{6}^{\prime}=\lambda^{6} c_{6}$ and $\Delta^{\prime}=\lambda^{12} \Delta$.

\section{Statement of global results}

Let $E$ be an elliptic curve defined over a number field $K$ with ring of integers $\mathcal{O}_{K}$ and class number $h_{K}$. We write $[n]$ for the multiplication-by- $n$ map on $E$.

Definition 2.1. An $n$-covering of $E$ is a pair $(C, \pi)$ where $C$ is smooth curve of genus one defined over $K$ and $\pi: C \rightarrow E$ is a morphism defined over $K$, such that $\pi=[n] \circ \psi$ for some isomorphism $\psi: C \cong E$ defined over $\bar{K}$. By abuse of notation we usually refer to the $n$-covering as $C$ rather than $(C, \pi)$.

Giving $C$ the structure of $n$-covering of its Jacobian is equivalent to specifying a $K$-rational divisor class $[D]$ on $C$ of degree $n$. Indeed given an $n$-covering $\pi: C \rightarrow E$ with $\pi=[n] \circ \psi$ we put $D=\psi^{*}\left(n .0_{E}\right)$. Conversely given $D$ we define $\pi: C \rightarrow$ $\operatorname{Pic}^{0} C=E ; P \mapsto[n . P-D]$. We say that an $n$-covering has trivial obstruction if it is possible to represent the given divisor class by a $K$-rational divisor $D$. In the cases $n=2,3$ this is the condition for the $n$-covering to have an equation of the form described in $\S 1$.

We are ready to state our main theorem.

Theorem 2.2. Let $E$ be an elliptic curve defined over a number field $K$, with $\mathcal{O}_{K}-$ coefficient Weierstrass equation

$$
y^{2}+a_{1} x y+a_{3} y=x^{3}+a_{2} x^{2}+a_{4} x+a_{6} .
$$

(i) Assume $\left(h_{K}, 2\right)=1$. If $C$ is an everywhere locally soluble 2-covering of $E$, then $C$ has an $\mathcal{O}_{K}$-coefficient equation

$$
y^{2}+\left(\alpha_{0} x^{2}+\alpha_{1} x+\alpha_{2}\right) y=a x^{4}+b x^{3}+c x^{2}+d x+e
$$

with the same invariants $c_{4}, c_{6}$ and $\Delta$ as (4).

(ii) Assume $\left(h_{K}, 3\right)=1$. If $C$ is an everywhere locally soluble 3-covering of $E$, then $C$ has an $\mathcal{O}_{K}$-coefficient equation

$$
a x^{3}+b y^{3}+c z^{3}+a_{2} x^{2} y+a_{3} x^{2} z+b_{1} x y^{2}+b_{3} y^{2} z+c_{1} x z^{2}+c_{2} y z^{2}+m x y z=0
$$

with the same invariants $c_{4}, c_{6}$ and $\Delta$ as (4). 
We give examples in Appendix B to show that the hypothesis on the class number cannot be removed.

Theorem 2.2 is most fruitfully applied in conjunction with the standard techniques for computing local and (where possible) global minimal Weierstrass equations. In the case $K=\mathbb{Q}$ we refer to $[4, \S 3.2]$. For $K$ a general number field one should consult the original papers of Kraus, Connell, Laska and Tate cited there. Tate's algorithm, which provides more detailed local information, is also described in [15]. A discussion of the passage from local to global in this context may be found in $[14$, Chapter VIII,, 8$]$.

Remark 2.3. By completing the square, Theorem 2.2(i) implies a weaker version where $\alpha_{0}=\alpha_{1}=\alpha_{2}=0$, but the binary quartic (5) has invariants $2^{4} c_{4}, 2^{6} c_{6}, 2^{12} \Delta$ for $c_{4}, c_{6}, \Delta$ the invariants of (4).

Remark 2.4. Theorem 2.2 constructs a model for $C$ with exactly the same invariants as (4). If our models for $C$ and $E$ have invariants $c_{4}^{\prime}, c_{6}^{\prime}, \Delta^{\prime}$ and $c_{4}, c_{6}, \Delta$ respectively, then an apparently weaker version would give that $\Delta^{\prime}$ divides $\Delta$ in $\mathcal{O}_{K}$. In fact the full result may be deduced from this. Indeed Theorem 1.1 gives $c_{4}^{\prime}=\lambda^{4} c_{4}, c_{6}^{\prime}=\lambda^{6} c_{6}$ and $\Delta^{\prime}=\lambda^{12} \Delta$ for some $\lambda \in K^{\times}$. So once we know $\lambda^{-1} \in \mathcal{O}_{K}$, we can reduce to the case $\lambda=1$ by rescaling, say, the $x$ co-ordinate of our model for $C$.

We recall a standard definition.

Definition 2.5. Let $E$ be an elliptic curve defined over a number field $K$. The minimal discriminant $\mathcal{D}_{E / K}$ is the integral ideal of $K$ generated by $\Delta\left(a_{1}, a_{2}, a_{3}, a_{4}, a_{6}\right)$ as

$$
y^{2}+a_{1} x y+a_{3} y=x^{3}+a_{2} x^{2}+a_{4} x+a_{6}
$$

ranges over all $\mathcal{O}_{K}$-coefficient Weierstrass equations for $E$.

The minimal discriminant $\mathcal{D}_{C / K}$ of a 2-covering or 3-covering $C$ with trivial obstruction is defined in an entirely analogous manner. The proof of Theorem 2.2 also gives

Theorem 2.6. Let $E$ be an elliptic curve defined over a number field $K$. Let $C$ be a 2-covering or 3-covering of $E$ with trivial obstruction.

(i) There is an integral ideal $\mathfrak{l}$ of $K$ such that $\mathcal{D}_{C / K}=\mathfrak{l}^{12} \mathcal{D}_{E / K}$.

(ii) If $C$ is everywhere locally soluble then $\mathfrak{l}=1$.

Remark 2.7. We suspect it may be possible to give a general definition of the minimal discriminant of a genus one curve $C$ that agrees with our definitions in the case $C$ is equipped with a $K$-rational divisor $D$ of degree $n=1,2$ or 3 . Theorem 2.6(ii) would then be a special case of the statement that if two genus one curves are everywhere locally isomorphic then they have the same minimal discriminant.

In order to compare Theorem 2.2 with earlier work on minimisation we combine it with Kraus' conditions for the existence of a Weierstrass equation with given invariants. In the case $K=\mathbb{Q}$ these state:

Theorem 2.8. Let $c_{4}, c_{6}$ be integers such that $\Delta=\left(c_{4}^{3}-c_{6}^{2}\right) / 1728$ is a non-zero integer. Then there exists an integer coefficient Weierstrass equation with invariants $c_{4}$ and $c_{6}$, if and only if

(i) $\operatorname{ord}_{3}\left(c_{6}\right) \neq 2$, and

(ii) either $c_{6} \equiv-1(\bmod 4)$, or $\operatorname{ord}_{2}\left(c_{4}\right) \geq 4$ and $c_{6} \equiv 0,8(\bmod 32)$. 
Proof. This is [9, Proposition 2]. In fact the necessity of these conditions is immediate from Tate's formulaire, i.e. (8) and (9).

Theorem 2.8 may be used to determine whether a given integer coefficient Weierstrass equation is minimal. Indeed a Weierstrass equation with invariants $c_{4}$ and $c_{6}$ is minimal at $p$ if and only if $p^{-4} c_{4}$ and $p^{-6} c_{6}$ fail to satisfy Kraus' conditions.

Definition 2.9. An integer coefficient binary quartic, respectively ternary cubic, with invariants $c_{4}$ and $c_{6}$ is $p$-reducible if it is equivalent to an integer coefficient binary quartic, respectively ternary cubic, with invariants $p^{-4} c_{4}$ and $p^{-6} c_{6}$.

Theorems 2.6 and 2.8 have the following corollary.

Corollary 2.10. Let $c_{4}$ and $c_{6}$ be integers such that $\Delta=\left(c_{4}^{3}-c_{6}^{2}\right) / 1728$ is a nonzero integer. The following conditions are necessary and sufficient for every integer coefficient p-adically soluble binary quartic, respectively ternary cubic, with invariants $c_{4}$ and $c_{6}$ to be $p$-reducible.

$$
\begin{aligned}
p \geq 5 & p^{4} \mid c_{4} \text { and } p^{6} \mid c_{6}, \\
p=3 & \text { either } 3^{5} \mid c_{4} \text { and } 3^{9} \mid c_{6}, \text { or } 3^{4}\left\|c_{4}, 3^{6}\right\| c_{6} \text { and } 3^{12} \mid \Delta, \\
p=2 & \text { either } 2^{8}\left|c_{4}, 2^{9}\right| c_{6} \text { and } 2^{-9} c_{6} \equiv 0,1(\bmod 4), \\
& \text { or } 2^{4}|| c_{4}, 2^{6} \| c_{6}, 2^{12} \mid \Delta \text { and } 2^{-6} c_{6} \equiv-1(\bmod 4) .
\end{aligned}
$$

The analogue of Corollary 2.10 for binary quartics without the cross terms (i.e. putting $\alpha_{0}=\alpha_{1}=\alpha_{2}=0$ ) is established by Cremona and Stoll [6, Appendix A] based on earlier work of Birch and Swinnerton-Dyer [3, Lemmas 3,4,5], [4, Proposition 3.6.1]. Their result is identical to ours at all primes $p \neq 2$, but is changed beyond recognition at $p=2$. The method of Birch and Swinnerton-Dyer has been extended to quadratic number fields by Cremona and Serf [5], [13]. In the case of ternary cubics, Corollary 2.10 is a theorem of Cremona and Stoll [7].

The method of Birch and Swinnerton-Dyer, and its generalisations cited above, require an analysis of a large number of tedious (yet elementary) special cases, especially when dealing with the primes $p=2,3$. As observed by Cremona and Stoll [6], [7] the results for $p \geq 5$ generalise immediately to an arbitrary number field. In contrast for $p=2,3$ it seems necessary to treat each possible value of the absolute ramification index as a new special case.

Our proof of Theorem 2.2 avoids all these special cases, and so gives a more general result. Nonetheless the old approach retains the following advantages:

- It gives efficient algorithms for minimising binary quartics and ternary cubics. This is useful in the number field method for 2-descent and 3-descent, where we obtain binary quartics and ternary cubics that initially have very large coefficients. An algorithm for minimising based on the proof of Theorem 2.2 would need to begin by finding explicit local solutions. It is therefore unlikely to be more efficient than the existing methods.

- In the invariant theory method for 2-descent (introduced in [3] and improved by Cremona in his program mwrank) it is more efficient to search for binary quartics without the cross terms. See [6] for a detailed discussion.

- As pointed out by Cremona and Stoll [6], [7] the hypothesis of local solubility may be weakened to that of solubility over an unramified extension of each local field. It is not clear how to match this result using our methods. 
It has long been known that Theorem 2.2 is false if one does not make the hypothesis that $C$ is everywhere locally soluble. Our favourite examples are given by the curves

$$
\begin{array}{lcc}
C_{a}: & y^{2}=a x^{4}+a^{3} & \Delta=2^{12} a^{12} \\
C_{a}: & x^{3}+a y^{3}+a^{2} z^{3}=0 & \Delta=-3^{9} a^{12} .
\end{array}
$$

One finds that for $p \geq 5$ a prime, $C_{p}$ has Jacobian $C_{1}$, yet $C_{p}$ is not $p$-reducible. Indeed if $C_{p}$ were $p$-reducible then one would obtain a model with good reduction at $p$. It would follow that $C_{p}\left(\mathbb{Q}_{p}\right) \neq \emptyset$ which, upon inspection of $(6)$, is plainly not the case.

It is easy to show that the converse of Theorem 2.6(ii) is false. For example the binary quartic $y^{2}=3 x^{4}+x^{2} z^{2}-z^{4}$ has discriminant $\Delta=2^{8} .3 .13^{2}$ yet is 2adically insoluble. Similarly the ternary cubic $x^{3}+2 y^{3}+5 z^{3}=0$ has discriminant $\Delta=-2^{4} .3^{9} \cdot 5^{4}$ yet is 3 -adically insoluble.

The following is an overview of the proofs of Theorems 2.2 and 2.6. First we reduce to a local statement, using strong approximation in the case of Theorem 2.2. Then we use our hypothesis of local solubility to rewrite our binary quartics and ternary cubics as equations of a particularly simple form. These special equations, which we call generalised Weierstrass equations, are introduced in $\S 4.2$. Our proofs are completed by enacting a subtle variant of Tate's algorithm to minimise generalised Weierstrass equations.

In a sequel to this paper we plan to generalise Theorem 2.2 to $n$-coverings of elliptic curves. The proof will be by induction on $n$ (in the local setting) starting from the case $n=3$ considered here. The case $n=4$ should be compared with Womack's algorithm [17] for minimising a pair of homogeneous quadratics in 4 variables.

It is clear that the models obtained in Theorem 2.2 are far from unique. An interesting problem would be to determine the number of integral equivalence classes of solutions. Here we say that two models are integrally equivalent if, in the notation of $\S 1.4$, they are related by an integer coefficient transformation with $\lambda$ a unit.

\section{The passage from local to global}

Let $K$ be a finite extension of $\mathbb{Q}_{p}$ with ring of integers $\mathcal{O}_{K}$. Theorem 2.2 has the following local analogue.

Theorem 3.1. Let $E$ be an elliptic curve defined over $K$. Let $c_{4}, c_{6}$ and $\Delta$ be the invariants of an $\mathcal{O}_{K}$-coefficient Weierstrass equation for $E$. Then

(i) Every soluble 2-covering $C$ of $E$ has an $\mathcal{O}_{K}$-coefficient equation

$$
y^{2}+\left(\alpha_{0} x^{2}+\alpha_{1} x+\alpha_{2}\right) y=a x^{4}+b x^{3}+c x^{2}+d x+e
$$

with invariants $c_{4}, c_{6}$ and $\Delta$.

(ii) Every soluble 3-covering $C$ of $E$ has an $\mathcal{O}_{K}$-coefficient equation

$$
a x^{3}+b y^{3}+c z^{3}+a_{2} x^{2} y+a_{3} x^{2} z+b_{1} x y^{2}+b_{3} y^{2} z+c_{1} x z^{2}+c_{2} y z^{2}+m x y z=0
$$

with invariants $c_{4}, c_{6}$ and $\Delta$.

We give the proof of Theorem 3.1 in $\S 4$. First we show how to deduce Theorems 2.2 and 2.6 from Theorem 3.1.

Proof of Theorem 2.6: Let $n=2$ or 3 . We recall that $E$ is an elliptic curve defined over a number field $K$, and that $C$ is an $n$-covering of $E$ with trivial obstruction. We 
fix a prime $\mathfrak{p}$ of $K$ and put $\gamma_{\mathfrak{p}}=\operatorname{ord}_{\mathfrak{p}}\left(\mathcal{D}_{C / K}\right), \varepsilon_{\mathfrak{p}}=\operatorname{ord}_{\mathfrak{p}}\left(\mathcal{D}_{E / K}\right)$. We aim to show

(i) $\gamma_{\mathfrak{p}}=\varepsilon_{\mathfrak{p}}+12 m$ for some integer $m \geq 0$, and

(ii) if $C\left(K_{\mathfrak{p}}\right) \neq \emptyset$ then $m=0$.

We choose an $\mathcal{O}_{K}$-coefficient model for $C$ whose discriminant $\Delta_{C}$ satisfies $\operatorname{ord}_{\mathfrak{p}}\left(\Delta_{C}\right)=$ $\gamma_{\mathfrak{p}}$, and an $\mathcal{O}_{K}$-coefficient Weierstrass equation for $E$ whose discriminant $\Delta_{E}$ satisfies $\operatorname{ord}_{\mathfrak{p}}\left(\Delta_{E}\right)=\varepsilon_{\mathfrak{p}}$.

To prove (i) we use the formulae of Appendix A to construct an $\mathcal{O}_{K}$-coefficient model for $E$ with discriminant $\Delta_{C}$. (Notice that for $\mathfrak{p} \nmid 6$ it suffices to use Theorem 1.1.) Thus $\varepsilon_{\mathfrak{p}} \leq \gamma_{\mathfrak{p}}$. Moreover $\Delta_{C}=\lambda^{12} \Delta_{E}$ for some $\lambda \in K^{\times}$. Putting $m=\operatorname{ord}_{\mathfrak{p}}(\lambda)$ gives $\gamma_{\mathfrak{p}}=\varepsilon_{\mathfrak{p}}+12 m$ as required.

To prove (ii) we use Theorem 3.1 to construct an $\mathcal{O}_{\mathfrak{p}}$-coefficient model for $C$ with discriminant $\Delta_{E}$. In the notation of $\S 1.4$ this new model is related to the old by a transformation $\left[\mu_{\mathfrak{p}}, r_{\mathfrak{p}}, A_{\mathfrak{p}}\right]$, respectively $\left[\mu_{\mathfrak{p}}, A_{\mathfrak{p}}\right]$. For $t \in K_{\mathfrak{p}}^{\times}$the following transformations are identical:

$$
\begin{aligned}
n=2 & {\left[\mu_{\mathfrak{p}}, r_{\mathfrak{p}}, A_{\mathfrak{p}}\right] } & =\left[t^{-2} \mu_{\mathfrak{p}}, t^{2} r_{\mathfrak{p}}, t A_{\mathfrak{p}}\right] \\
n=3 & {\left[\mu_{\mathfrak{p}}, A_{\mathfrak{p}}\right] } & =\left[t^{-3} \mu_{\mathfrak{p}}, t A_{\mathfrak{p}}\right] .
\end{aligned}
$$

We may therefore assume that $r_{\mathfrak{p}} \in \mathcal{O}_{\mathfrak{p}}^{3}$ and $A_{\mathfrak{p}} \in \operatorname{Mat}_{n}\left(\mathcal{O}_{\mathfrak{p}}\right)$. We approximate $r_{\mathfrak{p}}$ by a vector $r \in \mathcal{O}_{K}^{3}$ and $A_{\mathfrak{p}}$ by a matrix $A \in \operatorname{Mat}_{n}\left(\mathcal{O}_{K}\right)$. Since we can find a finite set of primes, not containing $\mathfrak{p}$, that generates the class group, there exists $\mu \in K^{\times}$ with $\operatorname{ord}_{\mathfrak{p}}(\mu)=\operatorname{ord}_{\mathfrak{p}}\left(\mu_{\mathfrak{p}}\right)$ yet $\operatorname{ord}_{\mathfrak{p}^{\prime}}(\mu) \geq 0$ for all $\mathfrak{p}^{\prime} \neq \mathfrak{p}$. Finally the transformation $[\mu, r, A]$, respectively $[\mu, A]$, gives a new $\mathcal{O}_{K}$-coefficient model for $C$ whose discriminant $\Delta$ satisfies $\operatorname{ord}_{\mathfrak{p}}(\Delta)=\varepsilon_{\mathfrak{p}}$. Thus $\gamma_{\mathfrak{p}} \leq \varepsilon_{\mathfrak{p}}$ as required.

Before proceeding with the proof of Theorem 2.2 we need two lemmas on strong approximation. For $A=\left(a_{i j}\right) \in \operatorname{Mat}_{n}\left(K_{\mathfrak{p}}\right)$ we put $\|A\|_{\mathfrak{p}}=\max _{1 \leq i, j \leq n}\left\|a_{i j}\right\|_{\mathfrak{p}}$.

Lemma 3.2. Let $S$ be a finite set of primes of $K$. Suppose we are given $A_{\mathfrak{p}} \in \mathrm{SL}_{n}\left(\mathcal{O}_{\mathfrak{p}}\right)$ for each prime $\mathfrak{p} \in S$ and let $\varepsilon>0$. Then there exists $A \in \mathrm{SL}_{n}\left(\mathcal{O}_{K}\right)$ such that $\left\|A-A_{\mathfrak{p}}\right\|_{\mathfrak{p}}<\varepsilon$ for all $\mathfrak{p} \in S$.

Proof. Let $E_{i j}$ be the $n \times n$ matrix with entry 1 in the $(i, j)$-th place, and zeros elsewhere. Using the identities

$$
\left(\begin{array}{ll}
1 & 1 \\
0 & 1
\end{array}\right)\left(\begin{array}{cc}
1 & 0 \\
-1 & 1
\end{array}\right)\left(\begin{array}{ll}
1 & 1 \\
0 & 1
\end{array}\right)=\left(\begin{array}{cc}
0 & 1 \\
-1 & 0
\end{array}\right)
$$

and

$$
\left(\begin{array}{cc}
1 & 0 \\
-\alpha^{-1} & 1
\end{array}\right)\left(\begin{array}{cc}
1 & \alpha-1 \\
0 & 1
\end{array}\right)\left(\begin{array}{ll}
1 & 0 \\
1 & 1
\end{array}\right)\left(\begin{array}{cc}
1 & \alpha^{-1}-1 \\
0 & 1
\end{array}\right)=\left(\begin{array}{cc}
\alpha & 0 \\
0 & \alpha^{-1}
\end{array}\right)
$$

one can show (via row and column operations) that $\operatorname{SL}_{n}\left(\mathcal{O}_{\mathfrak{p}}\right)$ is generated by the matrices $I_{n}+\lambda E_{i j}$ for $\lambda \in \mathcal{O}_{\mathfrak{p}}$ and $i \neq j$. So it suffices to treat the case $A_{\mathfrak{p}}=I_{n}+\lambda_{\mathfrak{p}} E_{i j}$ with $\lambda_{\mathfrak{p}}=0$ for all but one prime $\mathfrak{p}$ in $S$. We are done by the Chinese Remainder Theorem.

Lemma 3.3. Let $S$ be a finite set of primes of $K$ and let $\delta \in \mathcal{O}_{K}$. Suppose we are given $A_{\mathfrak{p}} \in \operatorname{Mat}_{n}\left(\mathcal{O}_{\mathfrak{p}}\right)$ with $\operatorname{det}\left(A_{\mathfrak{p}}\right)=\delta$ for each prime $\mathfrak{p} \in S$ and let $\varepsilon>0$. Then there exists $A \in \operatorname{Mat}_{n}\left(\mathcal{O}_{K}\right)$ with $\operatorname{det}(A)=\delta$ such that $\left\|A-A_{\mathfrak{p}}\right\|_{\mathfrak{p}}<\varepsilon$ for all $\mathfrak{p} \in S$. 
Proof. By Lemma 3.2 it suffices to treat the case where each local matrix $A_{\mathfrak{p}}$ is diagonal. We aim to construct a matrix $A$ with the required properties, say $A=\left(a_{i j}\right)$. Let $b \in \mathcal{O}_{K}$ be an $S$-unit which is $\mathfrak{p}$-adically small for all $\mathfrak{p} \in S$. (This is possible by the finiteness of the class group.) We set $a_{i} i+1=b$ for all $1 \leq i \leq n-1$. We then use the Chinese Remainder Theorem to choose diagonal entries for our matrix $A$ that are $\mathfrak{p}$-adically close to the diagonal entries of $A_{\mathfrak{p}}$ for all $\mathfrak{p} \in S$. We put

$$
a_{n 1}=(-1)^{n}\left(\prod_{i=1}^{n} a_{i i}-\delta\right) / b^{n-1} .
$$

Setting all remaining entries zero gives the required matrix $A$.

Proof of Theorem 2.2: Let $n=2$ or 3 . We recall that $E$ is an elliptic curve defined over a number field $K$, and $C$ is an everywhere locally soluble $n$-covering of $E$. In particular $C$ has trivial obstruction everywhere locally. It is shown in [11] that the obstruction takes values in the Brauer group. So by global class field theory, $C$ has trivial obstruction. (This is the only stage in the proof where we use the hypothesis of local solubility at the infinite places. This is only an issue for $n=2$.) Thus $C$ is represented by a binary quartic or a ternary cubic. We use the transformations of $\S 1.4$ to find such a model with $\mathcal{O}_{K}$-coefficients.

Let $\Delta_{C}$ and $\Delta_{E}$ be the discriminants of our models for $C$ and $E$. By Theorem 1.1 we have $\Delta_{C}=\lambda^{12} \Delta_{E}$ for some $\lambda \in K^{\times}$. Let $S$ be a finite set of primes containing a set of generators for the class group, and all primes $\mathfrak{p}$ with $\operatorname{ord}_{\mathfrak{p}}(\lambda) \neq 0$. Theorem 3.1 tells us that for each $\mathfrak{p} \in S$ our model for $C$ is equivalent to an $\mathcal{O}_{\mathfrak{p}}$-coefficient model with discriminant $\Delta_{E}$. In the notation of $\S 1.4$ the equivalence is given by a transformation $\left[\mu_{\mathfrak{p}}, r_{\mathfrak{p}}, A_{\mathfrak{p}}\right]$, respectively $\left[\mu_{\mathfrak{p}}, A_{\mathfrak{p}}\right]$, with $\mu_{\mathfrak{p}} \in K_{\mathfrak{p}}^{\times}, r_{\mathfrak{p}} \in K_{\mathfrak{p}}^{3}$ and $A_{\mathfrak{p}} \in \mathrm{GL}_{n}\left(K_{\mathfrak{p}}\right)$. We are free to make adjustments of the form (7). Not only does this allow us to assume that $r_{\mathfrak{p}} \in \mathcal{O}_{\mathfrak{p}}^{3}$ and $A_{\mathfrak{p}} \in \operatorname{Mat}_{n}\left(\mathcal{O}_{\mathfrak{p}}\right)$ for all $\mathfrak{p} \in S$, but also, since $\left(h_{K}, n\right)=1$, we can find $\mu \in K^{\times}$with

$$
\operatorname{ord}_{\mathfrak{p}}(\mu)= \begin{cases}\operatorname{ord}_{\mathfrak{p}}\left(\mu_{\mathfrak{p}}\right) & \text { for } \mathfrak{p} \in S, \\ 0 & \text { for } \mathfrak{p} \notin S .\end{cases}
$$

Our local transformations are now of the form $\left[\mu, r_{\mathfrak{p}}, A_{\mathfrak{p}}\right]$, respectively $\left[\mu, A_{\mathfrak{p}}\right]$ with $r_{\mathfrak{p}} \in \mathcal{O}_{\mathfrak{p}}^{3}$ and $A_{\mathfrak{p}} \in \operatorname{Mat}_{n}\left(\mathcal{O}_{\mathfrak{p}}\right)$.

Let $\lambda_{\mathfrak{p}}=\mu \operatorname{det} A_{\mathfrak{p}}$. Since $\Delta_{C}=\lambda_{\mathfrak{p}}^{12} \Delta_{E}$ it follows that $\lambda / \lambda_{\mathfrak{p}}$ is a root of unity. Making adjustments to $r_{\mathfrak{p}}$ and $A_{\mathfrak{p}}$ (say, by rescaling the $x$ co-ordinate on our local model) we may suppose that $\lambda=\lambda_{\mathfrak{p}}$ for all $\mathfrak{p} \in S$. We put $\delta=\lambda \mu^{-1}$. Then $\operatorname{det} A_{\mathfrak{p}}=\delta$ for all $\mathfrak{p} \in S$, and moreover $\delta \in \mathcal{O}_{K}$. We use Lemma 3.3 to construct a matrix $A \in \operatorname{Mat}_{n}\left(\mathcal{O}_{K}\right)$ with determinant $\delta$ that is $\mathfrak{p}$-adically close to $A_{\mathfrak{p}}$ for all $\mathfrak{p} \in S$. In the case $n=2$ we also approximate the $r_{\mathfrak{p}}$ for $\mathfrak{p} \in S$ by a vector $r \in \mathcal{O}_{K}^{3}$. Finally the transformation $[\mu, r, A]$, respectively $[\mu, A]$, gives a new $\mathcal{O}_{K}$-coefficient model for $C$ with discriminant $\Delta_{E}$. We are done by Remark 2.4 .

\section{Proofs in the local case}

We work over a local field $K$, complete with respect to a discrete valuation ord : $K^{\times} \rightarrow \mathbb{Z}$. We write $R$ for the ring of integers and $\pi$ for a uniformiser. The residue field is $k=R / \pi R$. We make no restriction on the characteristic of $k$ (or for that matter $K)$. 
4.1. Weierstrass equations. We recall some standard facts about elliptic curves and Weierstrass equations, many of which we have been using already. The formulae are taken from [14, Chapter III].

Definition 4.1. An elliptic curve $(E, 0)$ defined over $K$ is a smooth curve of genus one defined over $K$ equipped with a rational point $0 \in E(K)$.

We choose $x, y \in K(E)$ such that $\mathcal{L}(2.0)$ and $\mathcal{L}(3.0)$ have bases $1, x$ and $1, x, y$. Then the 7 elements $1, x, y, x^{2}, x y, x^{3}, y^{2}$ in the 6 dimensional vector space $\mathcal{L}(6.0)$ satisfy a linear dependence relation. Furthermore the coefficients of $x^{3}$ and $y^{2}$ are non-zero. Rescaling $x$ and $y$ we deduce that $(E, 0)$ has a Weierstrass equation

$$
y^{2}+a_{1} x y+a_{3} y=x^{3}+a_{2} x^{2}+a_{4} x+a_{6} .
$$

Following Tate's formulaire we define

$$
\begin{aligned}
& b_{2}=a_{1}^{2}+4 a_{2} \\
& b_{4}=2 a_{4}+a_{1} a_{3} \\
& b_{6}=a_{3}^{2}+4 a_{6} \\
& b_{8}=a_{1}^{2} a_{6}+4 a_{2} a_{6}-a_{1} a_{3} a_{4}+a_{2} a_{3}^{2}-a_{4}^{2}
\end{aligned}
$$

and

$$
\begin{aligned}
c_{4} & =b_{2}^{2}-24 b_{4} \\
c_{6} & =-b_{2}^{3}+36 b_{2} b_{4}-216 b_{6} \\
\Delta & =-b_{2}^{2} b_{8}-8 b_{4}^{3}-27 b_{6}^{2}+9 b_{2} b_{4} b_{6} .
\end{aligned}
$$

As noted in $\S 1$ these formulae for $c_{4}, c_{6}$ and $\Delta$ may also be obtained by specialising the invariants of a binary quartic or ternary cubic.

Definition 4.2. A Weierstrass equation for $(E, 0)$ is minimal if $\operatorname{ord}(\Delta)$ is minimal subject to the condition $a_{1}, a_{2}, a_{3}, a_{4}, a_{6} \in R$.

Any two Weierstrass equations for $(E, 0)$ are related by making a substitution of the form

$$
\begin{aligned}
& x=u^{2} x^{\prime}+r \\
& y=u^{3} y^{\prime}+u^{2} s x^{\prime}+t
\end{aligned}
$$

and then dividing through by $u^{6}$. This transformation is denoted $[u ; r, s, t]$. The coefficients $a_{i}^{\prime}$ of the new Weierstrass equation are related to the coefficients $a_{i}$ of the old via

$$
\begin{aligned}
u a_{1}^{\prime} & =a_{1}+2 s \\
u^{2} a_{2}^{\prime} & =a_{2}-s a_{1}+3 r-s^{2} \\
u^{3} a_{3}^{\prime} & =a_{3}+r a_{1}+2 t \\
u^{4} a_{4}^{\prime} & =a_{4}-s a_{3}+2 r a_{2}-(r s+t) a_{1}+3 r^{2}-2 s t \\
u^{6} a_{6}^{\prime} & =a_{6}+r a_{4}+r^{2} a_{2}+r^{3}-t a_{3}-t^{2}-r t a_{1} .
\end{aligned}
$$

The associated quantities (8) and (9) are transformed by

$$
\begin{aligned}
& u^{2} b_{2}^{\prime}=b_{2}+12 r \\
& u^{4} b_{4}^{\prime}=b_{4}+r b_{2}+6 r^{2} \\
& u^{6} b_{6}^{\prime}=b_{6}+2 r b_{4}+r^{2} b_{2}+4 r^{3} \\
& u^{8} b_{8}^{\prime}=b_{8}+3 r b_{6}+3 r^{2} b_{4}+r^{3} b_{2}+3 r^{4}
\end{aligned}
$$

and $u^{4} c_{4}^{\prime}=c_{4}, u^{6} c_{6}^{\prime}=c_{6}, u^{12} \Delta^{\prime}=\Delta$. 
4.2. Generalised Weierstrass equations. We generalise the standard definitions recalled in the last subsection.

Definition 4.3. A generalised elliptic curve $\left(E, 0,0^{\prime}\right)$ defined over $K$ is a smooth curve of genus one defined over $K$ equipped with an ordered pair of rational points $0,0^{\prime} \in E(K)$. The possibility $0=0^{\prime}$ is allowed.

We choose $x, y \in K(E)$ such that $\mathcal{L}\left(0+0^{\prime}\right)$ and $\mathcal{L}\left(2.0+0^{\prime}\right)$ have bases $1, x$ and $1, x, y$. Then the 8 elements $1, x, y, x^{2}, x y, x^{3}, y^{2}, x^{2} y$ in the 7 dimensional vector space $\mathcal{L}\left(4.0+3.0^{\prime}\right)$ satisfy a linear dependence relation. Furthermore the coefficient of $y^{2}$ is non-zero. We deduce that $\left(E, 0,0^{\prime}\right)$ has a generalised Weierstrass equation

$$
y^{2}+\alpha_{1} x y+\alpha_{3} y=\xi x^{2} y+\eta x^{3}+\alpha_{2} x^{2}+\alpha_{4} x+\alpha_{6} .
$$

The invariants $c_{4}, c_{6}$ and $\Delta$ are defined as polynomials in $\xi, \eta, \alpha_{1}, \alpha_{2}, \alpha_{3}, \alpha_{4}, \alpha_{6}$ by specialising the invariants of a ternary cubic.

Definition 4.4. A generalised Weierstrass equation for $\left(E, 0,0^{\prime}\right)$ is minimal if $\operatorname{ord}(\Delta)$ is minimal subject to the condition $\xi, \eta, \alpha_{1}, \alpha_{2}, \alpha_{3}, \alpha_{4}, \alpha_{6} \in R$.

Any two generalised Weierstrass equations for $\left(E, 0,0^{\prime}\right)$ are related by making a substitution of the form

$$
\begin{aligned}
& x=v^{2} w x^{\prime}+\rho \\
& y=v^{3} w^{2} y^{\prime}+v^{2} w \sigma x^{\prime}+\tau
\end{aligned}
$$

and then dividing through by $v^{6} w^{4}$. This transformation is denoted $[v, w ; \rho, \sigma, \tau]$. The coefficients of the new generalised Weierstrass equation are related to the old via

$$
\begin{aligned}
v^{-1} \xi^{\prime} & =\xi \\
w \eta^{\prime} & =\eta+\sigma \xi \\
v w \alpha_{1}^{\prime} & =\alpha_{1}-2 \rho \xi+2 \sigma \\
v^{2} w^{2} \alpha_{2}^{\prime} & =\alpha_{2}+(2 \rho \sigma+\tau) \xi-\sigma \alpha_{1}+3 \rho \eta-\sigma^{2} \\
v^{3} w^{2} \alpha_{3}^{\prime} & =\alpha_{3}-\rho^{2} \xi+\rho \alpha_{1}+2 \tau \\
v^{4} w^{3} \alpha_{4}^{\prime} & =\alpha_{4}+\rho(\rho \sigma+2 \tau) \xi-\sigma \alpha_{3}+2 \rho \alpha_{2}-(\rho \sigma+\tau) \alpha_{1}+3 \rho^{2} \eta-2 \sigma \tau \\
v^{6} w^{4} \alpha_{6}^{\prime} & =\alpha_{6}+\rho^{2} \tau \xi+\rho \alpha_{4}+\rho^{2} \alpha_{2}+\rho^{3} \eta-\tau \alpha_{3}-\tau^{2}-\rho \tau \alpha_{1} .
\end{aligned}
$$

We have arranged that if $\xi=0, \eta=1, \alpha_{i}=a_{i}$ and

$$
[v, w ; \rho, \sigma, \tau]=[u, 1 ; r, s, t]
$$

then these formulae reduce to those recalled in $\S 4.1$.

Proposition 4.5. Let $\left(E, 0,0^{\prime}\right)$ have generalised Weierstrass equation

$$
y^{2}+\alpha_{1} x y+\alpha_{3} y=\xi x^{2} y+\eta x^{3}+\alpha_{2} x^{2}+\alpha_{4} x+\alpha_{6} .
$$

Then $(E, 0)$ has Weierstrass equation

$$
Y^{2}+a_{1} X Y+a_{3} Y=X^{3}+a_{2} X^{2}+a_{4} X+a_{6}
$$

where

$$
\begin{aligned}
& a_{1}=\alpha_{1} \\
& a_{2}=\alpha_{2}+\xi \alpha_{3} \\
& a_{3}=\eta \alpha_{3}-\xi \alpha_{4} \\
& a_{4}=\eta \alpha_{4}+\xi \alpha_{2} \alpha_{3}-\xi^{2} \alpha_{6} \\
& a_{6}=\eta^{2} \alpha_{6}-\xi \eta \alpha_{1} \alpha_{6}+\xi \eta \alpha_{3} \alpha_{4}-\xi^{2} \alpha_{2} \alpha_{6} .
\end{aligned}
$$


Moreover the generalised Weierstrass equation (12) and its associated Weierstrass equation (13) have the same invariants $c_{4}, c_{6}$ and $\Delta$.

Proof. The required isomorphism is given by

$$
\begin{aligned}
X & =\eta x+\xi y \\
Y & =\eta y+\xi\left(-\alpha_{1} y+\xi x y+\eta x^{2}+\alpha_{2} x+\alpha_{4}\right) .
\end{aligned}
$$

The statement concerning invariants is proved by direct calculation. An alternative proof is given by specialising the formulae of Artin, Rodriguez-Villegas and Tate recalled in Appendix A.

Lemma 4.6. If we transform a generalised Weierstrass equation (12) by $[v, w ; \rho, \sigma, \tau]$ then the associated Weierstrass equation (13) is transformed by $[u ; r, s, t]$ where

$$
\begin{aligned}
u & =v w \\
r & =\rho \eta+\tau \xi \\
s & =\sigma-\rho \xi \\
t & =\tau \eta-2 \rho^{2} \xi \eta+\left(\rho \sigma \alpha_{1}-\rho \alpha_{2}+\sigma \alpha_{3}+2 \sigma \tau\right) \xi-\rho(\rho \sigma+\tau) \xi^{2} .
\end{aligned}
$$

Proof. A direct calculation.

4.3. An algorithm for minimising. We continue to write $R$ for the ring of integers of $K$. It is clear that every generalised Weierstrass equation is equivalent to one with coefficients in $R$.

Theorem 4.7. A generalised Weierstrass equation with coefficients in $R$ is minimal if and only if its associated Weierstrass equation is minimal.

Proof. It is clear from Proposition 4.5 that if a generalised Weierstrass equation is not minimal then its associated Weierstrass equation is not minimal. Indeed if the generalised Weierstrass equation is minimised by a transformation $[v, w ; \rho, \sigma, \tau]$ then its associated Weierstrass equation is minimised by $[u ; r, s, t]$ where $u, r, s, t$ are given by $(15)$.

To prove the theorem we give an algorithm for minimising a generalised Weierstrass equation, subject only to the hypothesis that the associated Weierstrass equation is not minimal. The basic idea is as follows. By hypothesis there is a transformation $[1 ; r, s, t]$ which when applied to the associated Weierstrass equation gives $\pi^{i} \mid a_{i}$ for all $i$. We hope to solve (15) for $\rho, \sigma, \tau$. If successful, we apply the transformation $[1,1 ; \rho, \sigma, \tau]$ to our generalised Weierstrass equation, and thus reduce to the case $\pi^{i} \mid a_{i}$ for all $i$. With a bit of luck we can then use (14) to show that the $\alpha_{i}$ are divisible by certain powers of $\pi$. Finally we minimise using either $[\pi, 1 ; 0,0,0]$ or $[1, \pi ; 0,0,0]$.

In practice our algorithm is a hybrid of the above sketch and the first few steps of Tate's algorithm: see [15].

Step 1 . If $\pi \mid \xi$ and $\pi \mid \eta$ then we repeatedly apply the transformation $\left[\pi^{-1}, \pi ; 0,0,0\right]$ until either $\xi$ or $\eta$ is a unit.

Step 2. By hypothesis $\pi \mid \Delta$. So the reduction $\bmod \pi$ of our generalised Weierstrass equation has a singular point. By Step 1 either $\pi \nmid \xi$ or $\pi \nmid \eta$. So the points at infinity on the reduction are smooth. Making a transformation of the form $[1,1 ; \rho, 0, \tau]$ we may suppose that the singular point is $(x, y)=(0,0)$. Then $\pi \mid \alpha_{3}, \alpha_{4}, \alpha_{6}$. By (14) we also have $\pi \mid a_{3}, a_{4}, a_{6}$. 
Step 3. By hypothesis $\pi \mid c_{4}=b_{2}^{2}-24 b_{4}$. But by Step 2 we already have $\pi \mid b_{4}=$ $2 a_{4}+a_{1} a_{3}$. Therefore $\pi \mid b_{2}$ where

$$
b_{2}=a_{1}^{2}+4 a_{2}=\alpha_{1}^{2}+4 \alpha_{2}+4 \xi \alpha_{3} .
$$

Since $\pi \mid \alpha_{3}$ we deduce $\pi \mid\left(\alpha_{1}^{2}+4 \alpha_{2}\right)$. Making a transformation of the form $[1,1 ; 0, \sigma, 0]$ we may assume that $\pi \mid \alpha_{i}$ for all $i$. By (14) we also have $\pi \mid a_{i}$ for all $i$.

Step 4. By hypothesis there is a transformation $[1 ; r, s, t]$ for which the transformed quantities $a_{i}^{\prime}$ satisfy $\pi^{i} \mid a_{i}^{\prime}$ for all $i$. Since we already have $\pi \mid a_{i}$ for all $i$ it follows by (10) that

$$
\pi|2 s, \quad \pi|\left(3 r-s^{2}\right), \quad \pi|2 t, \quad \pi|\left(3 r^{2}-2 s t\right), \quad \pi \mid\left(r^{3}-t^{2}\right) .
$$

From these we deduce $\pi \mid r, s, t$. (Recall that we make no assumption on the characteristic of $k$.)

The algorithm now splits into two cases.

Case $\pi \nmid \eta$. Making a transformation $[1, \eta ; 0,0,0]$ we may assume that $\eta=1$. Let $r, s, t$ be as in Step 4. We solve for $\rho, \sigma, \tau$ satisfying

$$
\begin{aligned}
& r=\rho+\xi \tau \\
& s=\sigma-\xi \rho \\
& t=\tau-2 \xi \rho^{2}+\xi\left(\alpha_{1} \rho \sigma-\alpha_{2} \rho+\alpha_{3} \sigma+2 \sigma \tau\right)-\xi^{2} \rho(\rho \sigma+\tau) .
\end{aligned}
$$

To do this we set $\left(\rho_{1}, \sigma_{1}, \tau_{1}\right)=(r, s, t)$ and recursively define

$$
\begin{aligned}
& \rho_{n}=r-\xi \tau_{n-1} \\
& \sigma_{n}=s+\xi \rho_{n} \\
& \tau_{n}=t+2 \xi \rho_{n}^{2}-\xi\left(\alpha_{1} \rho_{n} \sigma_{n}-\alpha_{2} \rho_{n}+\alpha_{3} \sigma_{n}+2 \sigma_{n} \tau_{n}\right)+\xi^{2} \rho_{n}\left(\rho_{n} \sigma_{n}+\tau_{n}\right) .
\end{aligned}
$$

Since $\pi \mid r, s, t$ and $\pi \mid \alpha_{1}, \alpha_{2}, \alpha_{3}$ it follows by induction that

$$
\begin{aligned}
& \rho_{n+1} \equiv \rho_{n} \quad\left(\bmod \pi^{n}\right) \\
& \sigma_{n+1} \equiv \sigma_{n} \quad\left(\bmod \pi^{n}\right) \\
& \tau_{n+1} \equiv \tau_{n} \quad\left(\bmod \pi^{n+1}\right) .
\end{aligned}
$$

Since $K$ is complete ${ }^{1}$ the sequences $\rho_{n}, \sigma_{n}, \tau_{n}$ converge to $\rho, \sigma, \tau \in R$, a solution to (16). We apply the transformation $[1,1 ; \rho, \sigma, \tau]$ and hence reduce to the case $\pi^{i} \mid a_{i}$ for all $i$. Since $\pi \mid \rho, \sigma, \tau$ we still have $\pi \nmid \eta$ and $\pi \mid \alpha_{i}$ for all $i$. We now use (14) to show that $\pi^{2} \mid \alpha_{6}$ since $\pi^{2} \mid a_{6}$, then $\pi^{2} \mid \alpha_{4}$ since $\pi^{2} \mid a_{4}$, then $\pi^{2} \mid \alpha_{3}$ since $\pi^{2} \mid a_{3}$, and so on. In the end we get $\pi^{i} \mid \alpha_{i}$ for all $i$. We can then minimise using the transformation $[\pi, 1 ; 0,0,0]$.

Case $\pi \mid \eta$. By Step 1 we have $\pi \nmid \xi$. Making a transformation $\left[\xi^{-1}, 1 ; 0,0,0\right]$ we may assume that $\xi=1$. Let $r, s, t$ be as in Step 4 . Since

$$
a_{4}^{\prime}=a_{4}-s a_{3}+2 r a_{2}-(r s+t) a_{1}+3 r^{2}-2 s t
$$

we have $\pi^{2} \mid a_{4}$. Then using (14) we obtain $\pi^{2} \mid \alpha_{6}$ and $\pi^{3} \mid a_{6}$. Since

$$
\begin{aligned}
& a_{3}^{\prime}=a_{3}+r a_{1}+2 t \\
& a_{6}^{\prime}=a_{6}+r a_{4}+r^{2} a_{2}+r^{3}-t a_{3}-t^{2}-r t a_{1}
\end{aligned}
$$

it follows that $\pi^{2} \mid t$ and $\pi^{2} \mid a_{3}$.

\footnotetext{
${ }^{1}$ In fact an approximate solution to (16) would suffice, so we don't really need that $K$ is complete.
} 
We apply the transformation $[1,1 ; 0, s, r]$ to our generalised Weierstrass equation. After this transformation we still have $\pi \mid \eta$ and $\pi \mid \alpha_{i}$ for all $i$. According to Lemma 4.6 the associated Weierstrass equation is transformed by $\left[1 ; r, s, t^{\prime}\right]$ where $t^{\prime}=r \eta+s \alpha_{3}+$ $2 r s$. Since $t \equiv t^{\prime} \equiv 0\left(\bmod \pi^{2}\right)$ it follows by $(10)$ and $(11)$ that

$$
\pi\left|a_{1}, \quad \pi^{2}\right| a_{2}, a_{3}, \quad \pi^{3}\left|a_{4}, \quad \pi^{4}\right| a_{6}
$$

and $\pi^{i} \mid b_{i}$ for all $i$. Using (14) we obtain $\pi^{2} \mid \alpha_{4}, \alpha_{6}$ and then

$$
\pi^{2}\left|\left(\alpha_{2}+\alpha_{3}\right), \quad \pi^{3}\right|\left(\alpha_{2} \alpha_{3}-\alpha_{6}\right), \quad \pi^{4} \mid \alpha_{2} \alpha_{6} .
$$

From these we deduce

$$
\pi\left|\alpha_{1}, \quad \pi^{2}\right| \alpha_{2}, \alpha_{3}, \alpha_{4}, \quad \pi^{3} \mid \alpha_{6}
$$

Since

$$
a_{6}=\eta^{2} \alpha_{6}-\eta \alpha_{1} \alpha_{6}+\eta \alpha_{3} \alpha_{4}-\alpha_{2} \alpha_{6}
$$

it follows that $\pi^{5} \mid a_{6}$. Next using

$$
\begin{aligned}
& b_{6}=a_{3}^{2}+4 a_{6} \\
& b_{8}=a_{1}^{2} a_{6}+4 a_{2} a_{6}-a_{1} a_{3} a_{4}+a_{2} a_{3}^{2}-a_{4}^{2}
\end{aligned}
$$

we obtain first $\pi^{3} \mid a_{3}$ and then $\pi^{4} \mid a_{4}$. By (14) we also have $\pi^{3} \mid \alpha_{4}$ and $\pi^{4} \mid \alpha_{6}$. We can then minimise using the transformation $[1, \pi ; 0,0,0]$.

Recall that we defined the invariants of a generalised Weierstrass equation (12) as the invariants of the ternary cubic

$$
y^{2} z+\alpha_{1} x y z+\alpha_{3} y z^{2}-\left(\xi x^{2} y+\eta x^{3}+\alpha_{2} x^{2} z+\alpha_{4} x z^{2}+\alpha_{6} z^{3}\right)=0 .
$$

A calculation shows that these are the same as the invariants of the binary quartic

$$
y^{2}+\left(-\xi x^{2}+\alpha_{1} x z+\alpha_{3} z^{2}\right) y=\eta x^{3} z+\alpha_{2} x^{2} z^{2}+\alpha_{4} x z^{3}+\alpha_{6} z^{4} .
$$

Proof of Theorem 3.1: Let $n=2$ or 3 . We recall that $E$ is an elliptic curve defined over $K$, with $K$ a finite extension of $\mathbb{Q}_{p}$. We are given an $\mathcal{O}_{K}$-coefficient Weierstrass equation for $E$ and a soluble $n$-covering $C$ of $E$. The structure of $n$ covering determines a $K$-rational divisor class $[D]$ on $C$. For 0 a rational point on $C$ the Riemann-Roch space $\mathcal{L}(D-(n-1) .0)$ is 1-dimensional. Hence

$$
D \sim(n-1) \cdot 0+0^{\prime}
$$

where $0^{\prime}$ is a rational point on $C$. By Theorem 4.7 the generalised elliptic curve $\left(C, 0,0^{\prime}\right)$ has an $\mathcal{O}_{K}$-coefficient generalised Weierstrass equation with the same invariants as a minimal Weierstrass equation for $E$. Then (18) or (17) is a model for the pair $(C,[D])$ with discriminant dividing that of our original Weierstrass equation. We are done by the local analogue of Remark 2.4.

Remark 4.8. More concretely, a soluble binary quartic, respectively ternary cubic, may be put in generalised Weierstrass form using (in the notation of §1.4) a transformation $[1,0, A]$, respectively $[1, A]$. Indeed in the case $n=2$ we move the rational point to $(x: z)=(1: 0)$. The resulting binary quartic has no $x^{4}$ term and is therefore of the form (18). In the case $n=3$ we move the rational point to $(x: y: z)=(0: 1: 0)$ and its tangent line to $z=0$. The resulting ternary cubic has no terms $y^{3}$ or $x y^{2}$ and is therefore of the form (17). We can then minimise by following the proof of Theorem 4.7. 


\section{Appendix A. Formulae for the Jacobian}

In $\S 1$ we defined the invariants $c_{4}, c_{6}$ and $\Delta$ of a binary quartic, respectively a ternary cubic. We now attempt to "work back" through the formulae (8) and (9) in order to define quantities $b_{2}, b_{4}, b_{6}, b_{8}$ and $a_{1}, a_{2}, a_{3}, a_{4}, a_{6}$. In the case $n=2$, we find

$$
c_{4} \equiv\left(\alpha_{1}^{2}-4 \alpha_{0} \alpha_{2}+4 c\right)^{2} \quad(\bmod 24) .
$$

We set $b_{2}=\alpha_{1}^{2}-4 \alpha_{0} \alpha_{2}+4 c$ and solve for $b_{4}, b_{6}, b_{8}$ using (9). Next we set $a_{1}=\alpha_{1}$ and $a_{2}=-\alpha_{0} \alpha_{2}+c$. It turns out that we can write $b_{4}=\alpha_{1} a_{3}+2 a_{4}$ where $a_{3}$ and $a_{4}$ are polynomials not involving $\alpha_{1}$. Putting $a_{6}=\left(b_{6}-a_{3}^{2}\right) / 4$ we obtain

$$
\begin{aligned}
& a_{1}=\alpha_{1} \\
& a_{2}=c-\alpha_{0} \alpha_{2} \\
& a_{3}=\alpha_{0} d+\alpha_{2} b \\
& a_{4}=-4 a e+b d-\left(\alpha_{0}^{2} e+\alpha_{0} \alpha_{2} c+\alpha_{2}^{2} a\right) \\
& a_{6}=-4 a c e+a d^{2}+b^{2} e-\left(\alpha_{0}^{2} c e+\alpha_{1}^{2} a e+\alpha_{2}^{2} a c+\alpha_{0} \alpha_{2} b d\right)+\alpha_{0} \alpha_{1} b e+\alpha_{1} \alpha_{2} a d .
\end{aligned}
$$

In the case $n=3$, we find

$$
c_{4} \equiv\left(m^{2}-4\left(a_{2} c_{2}+a_{3} b_{3}+b_{1} c_{1}\right)\right)^{2} \quad(\bmod 24) .
$$

We set $b_{2}=m^{2}-4\left(a_{2} c_{2}+a_{3} b_{3}+b_{1} c_{1}\right)$ and solve for $b_{4}, b_{6}, b_{8}$ using (9). Next we set $a_{1}=m$ and $a_{2}=-\left(a_{2} c_{2}+a_{3} b_{3}+b_{1} c_{1}\right)$. It turns out that we can write $b_{4}=m a_{3}+2 a_{4}$ where $a_{3}$ and $a_{4}$ are polynomials not involving $m$. Putting $a_{6}=\left(b_{6}-a_{3}^{2}\right) / 4$ we obtain

$$
\begin{array}{rl}
a_{1}=m & \\
a_{2}=- & \left(a_{2} c_{2}+a_{3} b_{3}+b_{1} c_{1}\right) \\
a_{3}=9 & a b c-\left(a b_{3} c_{2}+b a_{3} c_{1}+c a_{2} b_{1}\right)-\left(a_{2} b_{3} c_{1}+a_{3} b_{1} c_{2}\right) \\
a_{4}=- & 3\left(a b c_{1} c_{2}+a c b_{1} b_{3}+b c a_{2} a_{3}\right) \\
& +a\left(b_{1} c_{2}^{2}+b_{3}^{2} c_{1}\right)+b\left(a_{2} c_{1}^{2}+a_{3}^{2} c_{2}\right)+c\left(a_{2}^{2} b_{3}+a_{3} b_{1}^{2}\right) \\
& +a_{2} c_{2} a_{3} b_{3}+b_{1} c_{1} a_{2} c_{2}+a_{3} b_{3} b_{1} c_{1} \\
a_{6}=- & 27 a^{2} b^{2} c^{2}+9 a b c\left(a b_{3} c_{2}+c a_{2} b_{1}+b a_{3} c_{1}\right)+3 a b c\left(a_{2} b_{3} c_{1}+a_{3} b_{1} c_{2}\right) \\
& -\left(a^{2} b c_{2}^{3}+b^{2} c a_{3}^{3}+c^{2} a b_{1}^{3}+a^{2} c b_{3}^{3}+b^{2} a c_{1}^{3}+c^{2} b a_{2}^{3}\right) \\
& +2\left(a b c_{1} c_{2}+b c a_{2} a_{3}+c a b_{1} b_{3}\right)\left(a_{2} c_{2}+a_{3} b_{3}+b_{1} c_{1}\right) \\
& -3\left(a b a_{3} b_{3} c_{1} c_{2}+b c a_{2} a_{3} b_{1} c_{1}+c a a_{2} b_{1} b_{3} c_{2}\right) \\
& -\left(b_{1} c_{1}+a_{2} c_{2}\right)\left(a b_{3}^{2} c_{1}+b c_{2} a_{3}^{2}\right) \\
& -\left(c_{2} a_{2}+a_{3} b_{3}\right)\left(b c_{1}^{2} a_{2}+c a_{3} b_{1}^{2}\right) \\
& -\left(a_{3} b_{3}+b_{1} c_{1}\right)\left(c a_{2}^{2} b_{3}+a b_{1} c_{2}^{2}\right) \\
& -a_{2} a_{3} b_{1} b_{3} c_{1} c_{2}-3 a b c\left(a_{2} c_{2}+a_{3} b_{3}+b_{1} c_{1}\right) m \\
& +\left(a b\left(a_{3} c_{2}^{2}+b_{3} c_{1}^{2}\right)+b c\left(a_{2}^{2} c_{1}+a_{3}^{2} b_{1}\right)+a c\left(a_{2} b_{3}^{2}+b_{1}^{2} c_{2}\right)\right) m \\
& +\left(a b_{1} b_{3} c_{1} c_{2}+b a_{2} a_{3} c_{1} c_{2}+c a_{2} a_{3} b_{1} b_{3}\right) m \\
& -\left(a b c_{1} c_{2}+b c a_{2} a_{3}+c a b_{1} b_{3}\right) m^{2}+a b c m^{3} .
\end{array}
$$

We record an immediate consequence.

Lemma A.1. Let $a_{1}, a_{2}, a_{3}, a_{4}, a_{6}$ be the quantities associated to a binary quartic or ternary cubic, as defined above. Then the Weierstrass equation

$$
y^{2}+a_{1} x y+a_{3} y=x^{3}+a_{2} x^{2}+a_{4} x+a_{6}
$$

has the same invariants $c_{4}, c_{6}$ and $\Delta$ as the original binary quartic or ternary cubic. 
These formulae, in the case of a ternary cubic, are due to Artin, Rodriguez-Villegas and Tate [2]. Moreover it is shown in their paper that (19) is a formula for the Jacobian that works in arbitrary characteristic. In characteristic different from 2 and 3 this is already clear from Theorem 1.1.

\section{Appendix B. The class group}

We give some examples to show that the hypothesis on the class number in Theorem 2.2 cannot be removed. First we need two lemmas.

Lemma B.1. Let $\left[K: \mathbb{Q}_{p}\right]<\infty$ with $p \neq 2$. Let $[\mu, r, A]$ with $\mu \in K^{\times}, r \in K^{3}$ and $A \in \mathrm{GL}_{2}(K)$ be a transformation relating two $\mathcal{O}_{K}$-coefficient binary quartics with good reduction. Then

$$
\operatorname{ord}(\mu) \equiv \operatorname{ord}(\operatorname{det} A) \equiv 0 \quad(\bmod 2) .
$$

Proof. Since $p \neq 2$ we may assume the binary quartics have no cross terms (i.e. $\alpha_{0}=\alpha_{1}=\alpha_{2}=0$ ) and $r=0$. Let $\pi \in K$ with $\operatorname{ord}(\pi)=1$. Since both binary quartics have good reduction we have $\operatorname{ord}(\mu)=-\operatorname{ord}(\operatorname{det} A)$. So without loss of generality

$$
[\mu, r, A]=\left[\pi^{-a-b}, 0,\left(\begin{array}{cc}
\pi^{a} & 0 \\
0 & \pi^{b}
\end{array}\right)\right]
$$

for some $a \geq b$. If $a>b$ then the first binary quartic has singular reduction above $(x: z)=(0: 1)$. So $a=b$ and $\operatorname{ord}(\mu) \equiv \operatorname{ord}(\operatorname{det} A) \equiv 0(\bmod 2)$.

Lemma B.2. Let $\left[K: \mathbb{Q}_{p}\right]<\infty$. Let $[\mu, A]$ with $\mu \in K^{\times}$and $A \in \mathrm{GL}_{3}(K)$ be a transformation relating two $\mathcal{O}_{K}$-coefficient ternary cubics with good reduction. Then

$$
\operatorname{ord}(\mu) \equiv \operatorname{ord}(\operatorname{det} A) \equiv 0 \quad(\bmod 3) .
$$

Proof. Let $\pi \in K$ with $\operatorname{ord}(\pi)=1$. Since both ternary cubics have good reduction we have $\operatorname{ord}(\mu)=-\operatorname{ord}(\operatorname{det} A)$. So without loss of generality

$$
[\mu, A]=\left[\pi^{-a-b-c},\left(\begin{array}{ccc}
\pi^{a} & 0 & 0 \\
0 & \pi^{b} & 0 \\
0 & 0 & \pi^{c}
\end{array}\right)\right]
$$

for some $a \geq b \geq c$. If $b>c$ then the first ternary cubic has singular reduction at $(x: y: z)=(0: 0: 1)$. If $a>b=c$ then the reduction contains the line $x=0$. So $a=b=c$ and $\operatorname{ord}(\mu) \equiv \operatorname{ord}(\operatorname{det} A) \equiv 0(\bmod 3)$.

Example B.3. Let $K=\mathbb{Q}(\sqrt{-35})$ and $\alpha=(-1+\sqrt{-35}) / 2$. Let $E$ be the elliptic curve with Weierstrass equation

$$
y^{2}=x^{3}-11 x+14
$$

and $C$ the 2-covering of $E$ with equation

$$
y^{2}=\alpha\left(x^{2}-\bar{\alpha} z^{2}\right)\left(x^{2}-2 \bar{\alpha} z^{2}\right) .
$$

We have $\Delta_{E}=2^{9}$ and $\Delta_{C}=2^{9} .3^{12}$. The prime 3 factors in $\mathcal{O}_{K}$ as $(3)=\mathfrak{p} \overline{\mathfrak{p}}$ with $\mathfrak{p}^{2}=(\alpha)$. We can minimise (20) locally at $\mathfrak{p}$ and $\overline{\mathfrak{p}}$ by means of the transformations

$$
\left[3^{-1}, 0,\left(\begin{array}{ll}
1 & 0 \\
0 & 1
\end{array}\right)\right] \quad \text { and } \quad\left[1,0,\left(\begin{array}{cc}
1 & 0 \\
0 & 3^{-1}
\end{array}\right)\right]
$$


We suppose for a contradiction that $C$ has an $\mathcal{O}_{K}$-coefficient equation with the same invariants as $E$. Let $[\mu, r, A]$ be the transformation relating (20) and this new equation. Then by Lemma B.1 we find

$$
\begin{aligned}
& \operatorname{ord}_{\mathfrak{p}}(\mu) \equiv 1 \quad(\bmod 2) \\
& \operatorname{ord}_{\bar{p}}(\mu) \equiv 0 \quad(\bmod 2)
\end{aligned}
$$

and $\operatorname{ord}_{\mathfrak{q}}(\mu) \equiv 0(\bmod 2)$ for all $\mathfrak{q} \nmid 2,3$. Since 2 is inert in $K$ and $h_{K}=2$ it follows that $\mathfrak{p}$ is principal. This is the required contradiction. Finally we note that $\alpha \in\left(K_{2}^{*}\right)^{2}$, so $C$ is everywhere locally soluble.

Example B.4. Let $K=\mathbb{Q}(\sqrt{-23})$ and $\alpha=2+\sqrt{-23}$. Let $E$ be the elliptic curve with Weierstrass equation

$$
y^{2}+y=x^{3}+(2 \sqrt{-23}) x+(8+3 \sqrt{-23})
$$

and $C$ the 3 -covering of $E$ with equation

$$
3 x^{3}+\alpha\left(x^{2} y-x z^{2}+3 y^{2} z\right)+\alpha^{2} y^{3}=0 .
$$

We have $\left(\Delta_{E}\right)=\mathfrak{p}_{1} \mathfrak{p}_{2}$ and $\Delta_{C}=\alpha^{12} \Delta_{E}$ where $\mathfrak{p}_{1}=(1+6 \sqrt{-23})$ and $\mathfrak{p}_{2}=(1527+$ $446 \sqrt{-23})$ are prime ideals. The prime 3 factors in $\mathcal{O}_{K}$ as $(3)=\mathfrak{p} \overline{\mathfrak{p}}$ with $\mathfrak{p}^{3}=(\alpha)$. We can minimise (21) locally at $\mathfrak{p}$ by means of the transformation

$$
\left[3^{-4},\left(\begin{array}{ccc}
3 & 0 & 0 \\
0 & 1 & 0 \\
0 & 0 & 1
\end{array}\right)\right] .
$$

We suppose for a contradiction that $C$ has an $\mathcal{O}_{K}$-coefficient equation with the same invariants as $E$. Let $[\mu, A]$ be the transformation relating (21) and this new equation. Then by Lemma B.2 we find

$$
\operatorname{ord}_{\mathfrak{p}}(\mu) \equiv 2 \quad(\bmod 3)
$$

and $\operatorname{ord}_{\mathfrak{q}}(\mu) \equiv 0(\bmod 3)$ for all $\mathfrak{q} \neq \mathfrak{p}, \mathfrak{p}_{1}, \mathfrak{p}_{2}$. Since $\mathfrak{p}_{1}$ and $\mathfrak{p}_{2}$ are principal and $h_{K}=3$ it follows that $\mathfrak{p}$ is principal. This is the required contradiction. Finally we note that $C$ is globally soluble.

\section{Acknowledgements}

I would like to thank John Cremona and Michael Stoll for sharing many of their ideas on this topic. The computer calculations in support of this work were performed using MAGMA [10].

\section{References}

[1] S.Y. An, S.Y. Kim, D.C. Marshall, S.H. Marshall, W.G. McCallum and A.R. Perlis, Jacobians of genus one curves, J. Number Theory 90 (2001), no. 2, 304-315.

[2] M. Artin, F. Rodriguez-Villegas, J. Tate, On the Jacobians of plane cubics, Adv. Math. 198 (2005), no. 1, 366-382.

[3] B.J. Birch and H.P.F. Swinnerton-Dyer, Notes on elliptic curves I. J. Reine Angew. Math. 212 (1963), 7-25.

[4] J.E. Cremona, Algorithms for modular elliptic curves, Second edition, Cambridge University Press, Cambridge, 1997.

[5] J.E. Cremona and P. Serf, Computing the rank of elliptic curves over real quadratic number fields of class number 1, Math. Comp. 68 (1999), no. 227, 1187-1200. 
[6] J.E. Cremona and M. Stoll, Minimal models for 2-coverings of elliptic curves, LMS J. Comput. Math. 5 (2002), 220-243.

[7] J.E. Cremona and M. Stoll, Minimisation and reduction for 3- and 4-coverings of elliptic curves, in preparation.

[8] D. Hilbert, Theory of algebraic invariants, Cambridge University Press, Cambridge, 1993.

[9] A. Kraus, Quelques remarques à propos des invariants $c_{4}, c_{6}$ et $\Delta$ d'une courbe elliptique. Acta Arith. 54 (1989), no. 1, 75-80.

[10] MAGMA is described in W. Bosma, J. Cannon and C. Playoust, The Magma algebra system I: The user language, J. Symb. Comb. 24 (1997), 235-265. (See also the Magma home page at http://magma.maths. usyd.edu.au/magma/.)

[11] C. O'Neil, The period-index obstruction for elliptic curves. J. Number Theory 95 (2002), no. 2, 329-339.

[12] G. Salmon, A treatise on the higher plane curves, Third edition, Hodges, Foster and Figgis, Dublin, 1879.

[13] P. Serf, The rank of elliptic curves over real quadratic number fields of class number $1, \mathrm{PhD}$ thesis, Universität des Saarlandes, Saarbrücken, 1995.

[14] J.H. Silverman, The arithmetic of elliptic curves, Graduate Texts in Mathematics 106, SpringerVerlag, New York, 1986.

[15] J.H. Silverman, Advanced topics in the arithmetic of elliptic curves, Graduate Texts in Mathematics 151, Springer-Verlag, New York, 1994.

[16] A. Weil, Remarques sur un mémoire d'Hermite, Arch. Math. 5 (1954), 197-202.

[17] T. Womack, Explicit descent on elliptic curves, PhD thesis, University of Nottingham, 2003.

University of Cambridge, DPMms, Centre for Mathematical Sciences, Wilberforce Road, Cambridge CB3 0WB, UK

E-mail address: T.A.Fisher@dpmms.cam.ac.uk 